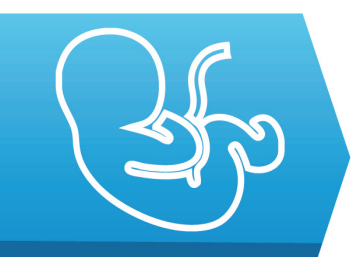

EMBRYOLOGY

\title{
Early development of human ganglionic eminences assessed in vitro by using 7.04 Tesla micro-MRI - a pilot study
}

Dan Boitor-Borza ${ }^{1}$, Flavius Turcu ${ }^{2}$, Stefan Farcasanu ${ }^{2}$, Carmen Crivii $^{1}$

1) Department of Anatomy, Iuliu Hatieganu University of Medicine and Pharmacy, Cluj-Napoca, Romania

2) Faculty of Physics, National Centre of Magnetic Resonance, "BabeşBolyai” University, Cluj-Napoca, Romania
DOI: $10.15386 / \mathrm{mpr}-1715$

Manuscript received: 10.05 .2020

Received in revised form: 21.06 .2020

Accepted: 07.07.2020

Address for correspondence:

danboitor@yahoo.com

This work is licensed under a Creative Commons Attribution-NonCommercialNoDerivatives 4.0 International License

\begin{abstract}
Background and aims. Ganglionic eminences are temporary structures which appear during the $5^{\text {th }}$ week post-fertilization on the floor of telencephalic vesicles and disappear until the $35^{\text {th }}$ week of gestation. The aim of this descriptive study of morphological research is to depict the ganglionic eminences within the embryonic and early fetal brains by using micro-MRI.

Methods. Six human embryos and fetuses ranging from $21 \mathrm{~mm}$ crown-rump length CRL ( 9 gestational week $\mathrm{GW}$ ) to $85 \mathrm{~mm}$ CRL (14 GW) were examined in vitro by micro-MRI. The investigation was performed with a Bruker BioSpec 70/16USR scanner (Bruker BioSpin MRI GmbH, Ettlingen, Germany) operating at 7.04 Tesla.

Results. We describe the morphological characteristics of the ganglionic eminences at different gestational ages. The acquisition parameters were modified for each subject in order to obtain an increased spatial resolution. The remarkable spatial resolution of $27 \mu \mathrm{m} / \mathrm{voxel}$ allows visualization of millimetric structures of the developing brain on high quality micro-MR images.

Conclusion. In our study we give the description of the ganglionic eminences within the embryonic and early fetal brains by using micro-MRI, which, to the best of our knowledge, have not been previously documented in literature. Micro-MRI provides accurate images, which are comparable with the histological slices.
\end{abstract}

Keywords: micro-MRI, ganglionic eminences, brain, fetal development

\section{Background and aims}

Ganglionic eminences (GE) are subcortical structures of gray matter which appear during the $5^{\text {th }}$ week postfertilization (Carnegie stages CS 14 and 15 ) on the floor of telencephalic vesicles (subpallium) [1]. They are also called ventricular eminences as they bulge into the primitive lateral ventricles. These structures give rise to basal ganglia, thalamic and olfactory interneurons, as well as cortical GABAergic interneurons. The latter migrate tangentially towards the cortical plate of the telencephalic vesicles to become interneurons in the neocortex, hyppocampus and globus pallidus [2].

GE are temporary structures, as they disappear by the $35^{\text {th }}$ week of gestation. Müller and O'Rahilly [3] have shown that in fact they consist of two distinct parts with different origins: medial ventricular eminence (MVE) of diencephalic origin and lateral ventricular eminence (LVE) of telencephalic origin. The caudal undivided portion of the ganglionic eminence (CGE) give rise to the amygdaloid nucleus $[4,5,6]$. GE distinguish by their large volume and by the exceptional persistence of the subventricular layer [7].

The contribution of micro-MRI to the morphological assessment of human embryos has already been presented in literature. It is proved that this method is effective and reproducible. Also, it can provide clear images due to a remarkable spatial resolution and high tissue contrast, 
comparable with histological sections [8]. Micro-MRI is also known as "microscopy MRI" or "histology MRI" because of the remarkable spatial resolution of $24 \mu \mathrm{m} /$ voxel [9]. Smith et al. [10] used for the first time this method in studies on human or non-human embryos, showing that micro-MRI is feasible for in vitro studies on human embryos, since the spatial resolution is about $20 \mu \mathrm{m} /$ voxel in chemically fixed specimens. Despite this, morphological studies on chemically fixed human embryos using micro-MRI are rare $[8,11,12]$. Previous experiments that we conducted showed that it was impossible to identify the GE by micro-MRI earlier than $9 \mathrm{GW}$ or $21 \mathrm{~mm}$ CRL [13].

The aim of this observational descriptive study of morphological research is to depict the GE within the embryonic and early fetal brains, which, to the best of our knowledge, have not been previously documented in literature by using micro-MRI.

\section{Methods}

\section{Human embryos and fetuses}

Six embryos and fetuses ranging from $21 \mathrm{~mm}$ CRL (9 gestational weeks GW) to $85 \mathrm{~mm}$ CRL (14 GW) were examined in vitro by using 7.04 Tesla micro-MRI. They belong to the collection of the Department of Anatomy and Embryology of Iuliu Hatieganu University of Medicine and Pharmacy of Cluj-Napoca and are approximately 8-10 years old. The subjects were collected from legally induced abortions. They were preserved in a $9 \%$ formalin solution during the first 48 hours at $4^{\circ} \mathrm{C}$, and were subsequently stored at room temperature. The study protocol was approved by the Ethics Committee of Iuliu Hatieganu University of Medicine and Pharmacy of ClujNapoca, Romania.

The embryos were morphologically examined in order to classify them in a Carnegie stage according to the criteria established by O'Rahilly and Müller [14]. Inclusion criteria were: excellent quality of the tissues and normal external morphology.

The gestational age was calculated from the crownrump length (CRL) of the specimens and expressed in weeks of gestation (completed weeks from the last menstrual period) (Table I).

The micro-MRI images were compared with anatomical and histological reference images found in classical books of embryology and histology [15].

Table I. Characteristics of subjects.

\begin{tabular}{|l|c|c|c|c|c|c|} 
Subject & $\mathbf{1}$ & $\mathbf{2}$ & $\mathbf{3}$ & $\mathbf{4}$ & $\mathbf{5}$ & $\mathbf{6}$ \\
\hline GW & 9 & 10 & 11 & 12 & 13 & 14 \\
CRL (mm) & 21 & 32 & 38 & 55 & 66 & 85 \\
CS & 20 & 23 & & & &
\end{tabular}

GW: gestational weeks, CRL: crown-rump length, CS: Carnegie stage

\section{Acquisition of images}

MRI investigation was performed by using a Bruker BioSpec 70/16USR scanner (Bruker BioSpin MRI GmbH, Ettlingen, Germany) operating at 7.04 Tesla. The study was conducted at the National Center of MRI, Faculty of Physics of "Babes-Bolyai" University of Cluj-Napoca.

The BGS 9S HP gradient unit is capable to generate a maximum value of $760 \mathrm{mT} / \mathrm{m}$ across the $72 \mathrm{~mm}$ active radio frequency (RF) coil space. A $40 \mathrm{~mm}$ Quadrature Volume Coil (QVC) for RF excitation and signal acquisition was used.

All the experiments were performed at room temperature $\left(20^{\circ} \mathrm{C}\right)$. The specimens were immersed in a $9 \%$ formaldehyde solution inside plastic cylindrical containers. The adjustments of the anatomical references were obtained by $\mathrm{T} 2$-weighted images, acquired in all three orthogonal planes.

The processing of $2 \mathrm{D}$ acquired images was made with ParaVision ${ }^{\circledR}$ software (Bruker Corporation, Ettlingen, Germany).

The protocol FISP-3D was used for all experiments. The acquisition parameters were modified for each subject in order to obtain the highest possible resolution (Table II). For the same reason different matrices were overlapped over various fields of view according to the length of the subjects.

The most significant images were selected. In the T1weighted images protocol the GE provide a hyperintense signal.

Table II. Parameters of acquisition for each subject.

\begin{tabular}{|c|c|c|c|c|c|c|c|c|c|}
\hline Subject & $\begin{array}{l}\text { FOV } \\
(\mathrm{cm})\end{array}$ & $\begin{array}{l}\text { Matrix } \\
\text { (pixel) }\end{array}$ & slices & $\begin{array}{l}\text { Resolution } \\
\text { (cm/voxel) }\end{array}$ & $\begin{array}{c}\mathrm{TE} \\
(\mathrm{ms})\end{array}$ & $\begin{array}{c}\text { TR } \\
(\mathrm{ms})\end{array}$ & $\begin{array}{c}\text { FA } \\
\text { (grade) }\end{array}$ & $\begin{array}{c}\text { Bandwidth } \\
(\mathrm{Hz})\end{array}$ & $\mathbf{N A}$ \\
\hline 1 & $3.06 \times 2.90 \times 2.5$ & $512 \times 512 \times 256$ & 256 & $0.0060 \times 0.0057 \times 0.0098$ & 6.3 & 12.7 & 15 & 50000 & 70 \\
\hline 2 & $2.56 \times 2.50 \times 4$ & $512 \times 512 \times 256$ & 256 & $0.005 \times 0.0049 \times 0.0156$ & 6.4 & 12.8 & 15 & 50000 & 60 \\
\hline 3 & $1.86 \times 1.70 \times 3$ & $512 \times 384 \times 256$ & 256 & $0.0036 \times 0.0044 \times 0.0117$ & 6.7 & 18 & 15 & 50000 & 94 \\
\hline 4 & $4.76 \times 2.90 \times 3$ & $720 \times 512 \times 256$ & 256 & $0.0066 \times 0.0057 \times 0.0117$ & 8.4 & 16.8 & 15 & 50000 & 47 \\
\hline 5 & $4.96 \times 2.90 \times 2.80$ & $512 \times 512 \times 256$ & 256 & $0.0097 \times 0.0057 \times 0.0109$ & 6.3 & 12.7 & 15 & 50000 & 80 \\
\hline 6 & $5.16 \times 5.20 \times 4.70$ & $720 \times 512 \times 256$ & 256 & $0.0072 \times 0.0102 \times 0.0184$ & 8.3 & 16.6 & 15 & 50000 & 52 \\
\hline
\end{tabular}

FOV: field of view, TE: echo time, TR: repetition time, FA: flip angle, NA: number of acquisitions 


\section{Results}

The GE are visible in a $21 \mathrm{~mm}$ CRL embryo (Figure 1). They appear as an obvious thickening of the ventral wall of the telencephalic vesicles (TV), the socalled subpallium, while the dorsal wall is very thin. In this stage the GE consist of two distinct masses: MVE and LVE located ventrally and caudally from a large interventricular foramen which is occupied by the choroid plexus of the future lateral ventricle. The two masses are separated by a longitudinal sulcus which is clearly seen. In this stage the lamination of the subpallium can already be noticed. The GE lies over o thickening of the subpallium where the basal nuclei develop.

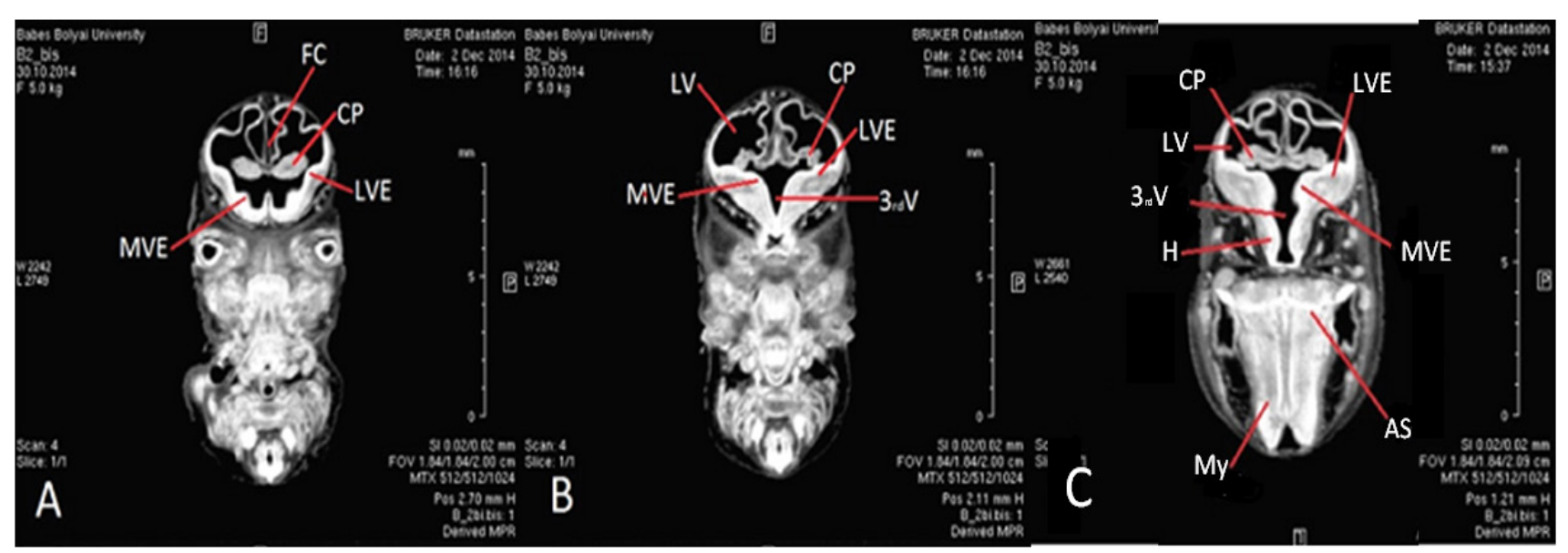

Figure 1. Human embryo $21 \mathrm{~mm}$ CRL, $9 \mathrm{GW}$, CS 20. Coronal/frontal (A, B) and axial/transversal (C) sections are shown. MVE: medial ventricular eminence, LVE: lateral ventricular eminence, CP: choroid plexus, FC: falx cerebri, LV: lateral ventricle, 3rdV : third ventricle, My: myelencephalon, AS : acoustic striae, H: hypothalamus.

In a $32 \mathrm{~mm}$ CRL embryo the GE are well developed. The MVE bulges into the third ventricle and the LVE bulges into the lateral ventricle. Those two portions are well defined and separated by a sulcus which is clearly shown in figure 2. They have similar dimensions. Furthermore, the
GE is clearly delimitated from the lateral and medial walls of the TV by two deep longitudinal sulci. In this embryo we can observe for the first time the nucleus accumbens which develops in the rostro-medial portion of the subpallium as part of the basal striatum.

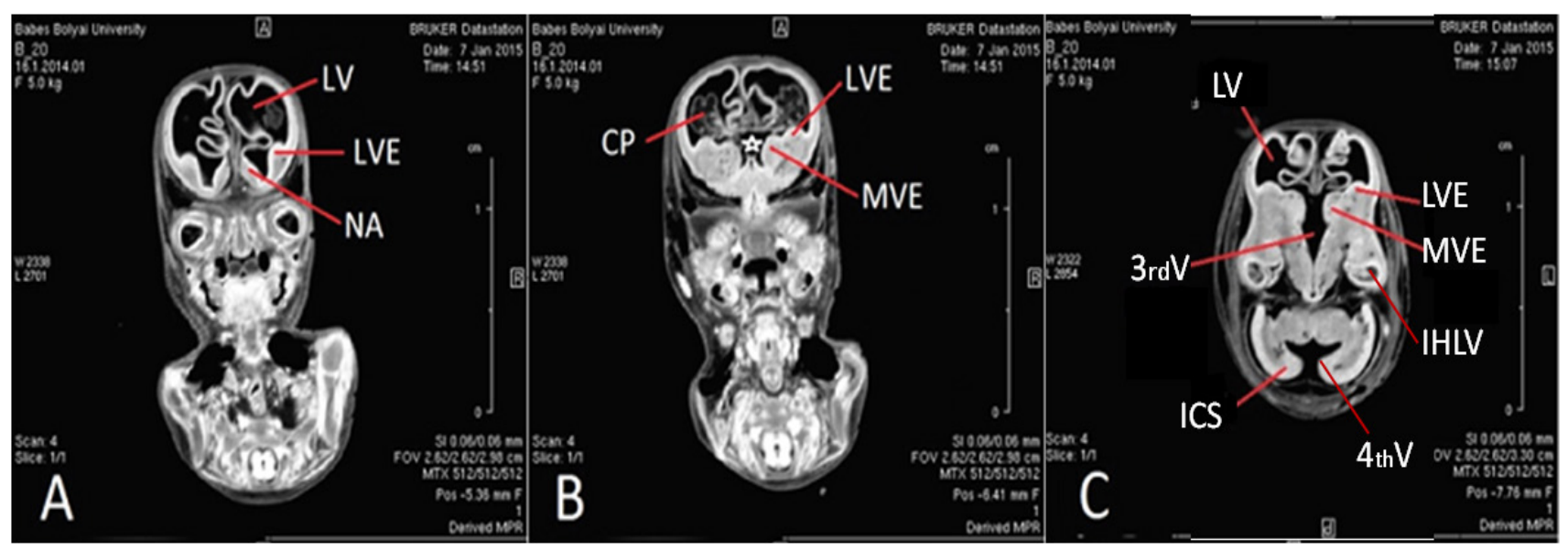

Figure 2. Human embryo $32 \mathrm{~mm}$ CRL, $10 \mathrm{GW}$, CS 23. Coronal (A, B) and axial (C) slices are presented. LV: lateral ventricle, LVE: lateral ventricular eminence, NA: nucleus accumbens, CP: choroid plexus, 3rdV and star in (B): third ventricle, ICS: internal cerebellar swelling, IHLV: inferior horn of the lateral ventricle, 4 thV: fourth ventricle. 
The sulcus between MVE and LVE can still be distinguished in a $38 \mathrm{~mm}$ CRL fetus (Figure 3B) but only at the rostral portion which lies near the nucleus accumbens. The MVE is separated from the nucleus accumbens by a deep fissure which is in fact the sulcus terminalis. In the caudal portion located laterally to the thalamus the limit between the MVE and LVE is no longer observed, as the thalamus is strongly developed in the lateral wall of the $3^{\text {rd }}$ ventricle (Figure 3C). The dorsal wall of the TV is still very thin while the lateral wall adjacent to the GE becomes thicker.

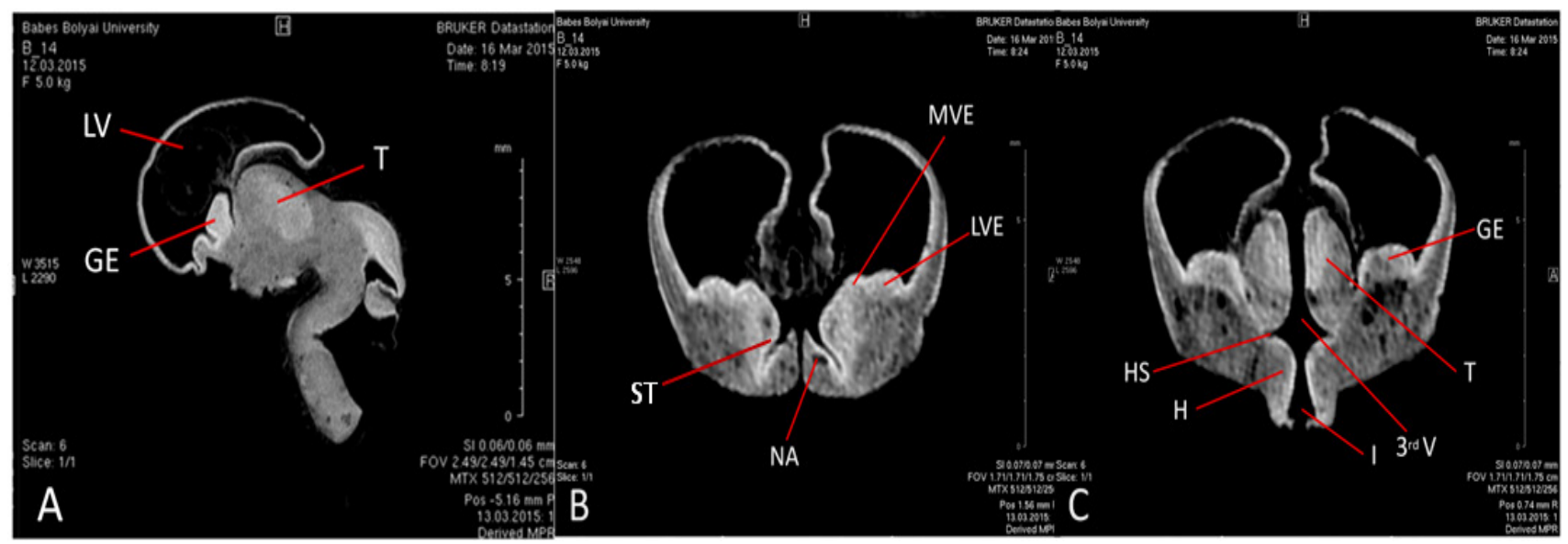

Figure 3. Human fetus $38 \mathrm{~mm}$ CRL, $11 \mathrm{GW}$. Sagittal (A) and coronal (B, C) sections are shown. LV: lateral ventricle, GE: ganglionic eminence, T: thalamus, MVE: medial ventricular eminence, LVE: lateral ventricular eminence, NA: nucleus accumbens, HS: hypothalamic sulcus, H: hypothalamus, I: infundibulum, 3rdV: third ventricle, ST: sulcus terminalis.

The GE is well depicted in a $55 \mathrm{~mm}$ CRL fetus as it lies dorsally to the basal ganglia and rostro-laterally to the thalamus. The internal capsule is clearly seen and separates the GE from the lenticular nucleus. The two portions of the lenticular nucleus, putamen and globus pallidus are noticed (Figure 4). The limit between the MVE and LVE is imprecise even in the most rostral part of the GE. It looks like the MVE diminishes while the LVE strongly develops. In the rostral portion the GE is wide while the CGE that is located laterally to the thalamus is narrow.

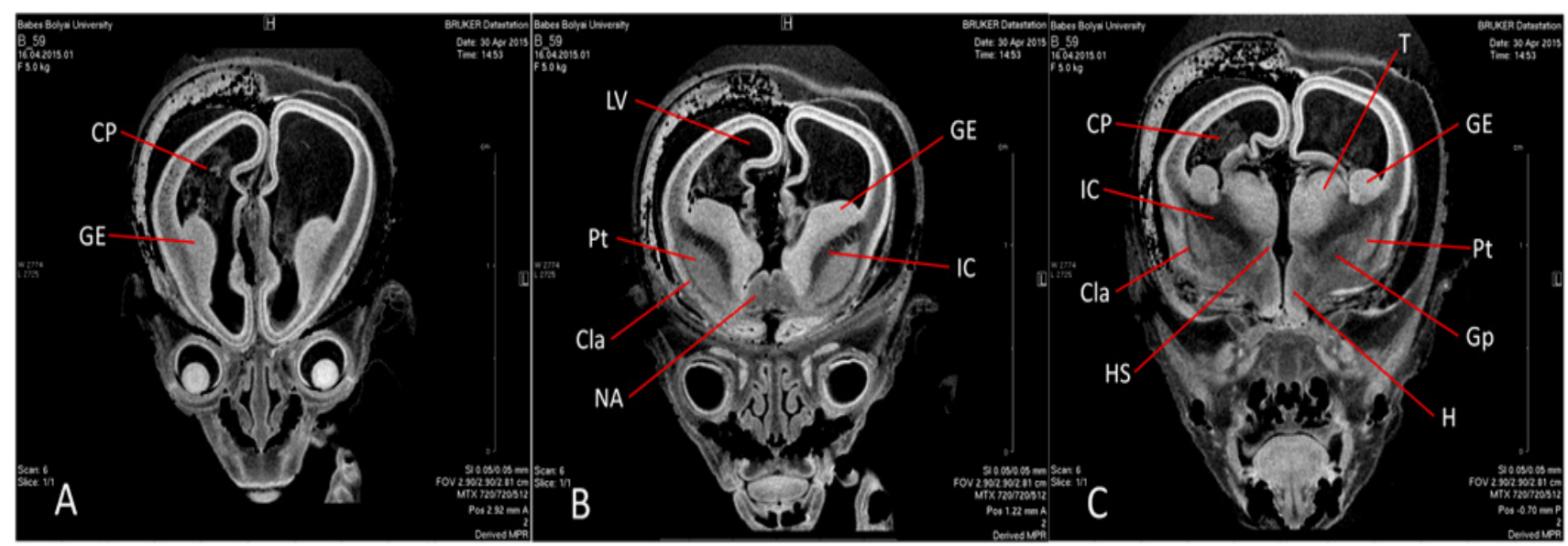

Figure 4. Human fetus $55 \mathrm{~mm} \mathrm{CRL}, 12 \mathrm{GW}$. Coronal sections (A, B, C) are presented. GE: ganglionic eminence, CP: choroid plexus, LV: lateral ventricle, Pt: putamen, Cla: claustrum, NA: nucleus accumbens, IC: internal capsule, HS: hypothalamic sulcus, H: hypothalamus, Gp: globus pallidus, T: thalamus. 
In a $66 \mathrm{~mm} \mathrm{CRL}$ fetus the GE is more developed in the rostral part of the brain, where it will give rise to the caudate nucleus (Figure 5). The limit between the MVE and LVE is no longer visible and the GE looks like a single voluminous mass providing a hyperintense signal in T1-wi protocol. In the caudal portion the GE is narrower than in the rostral portion.

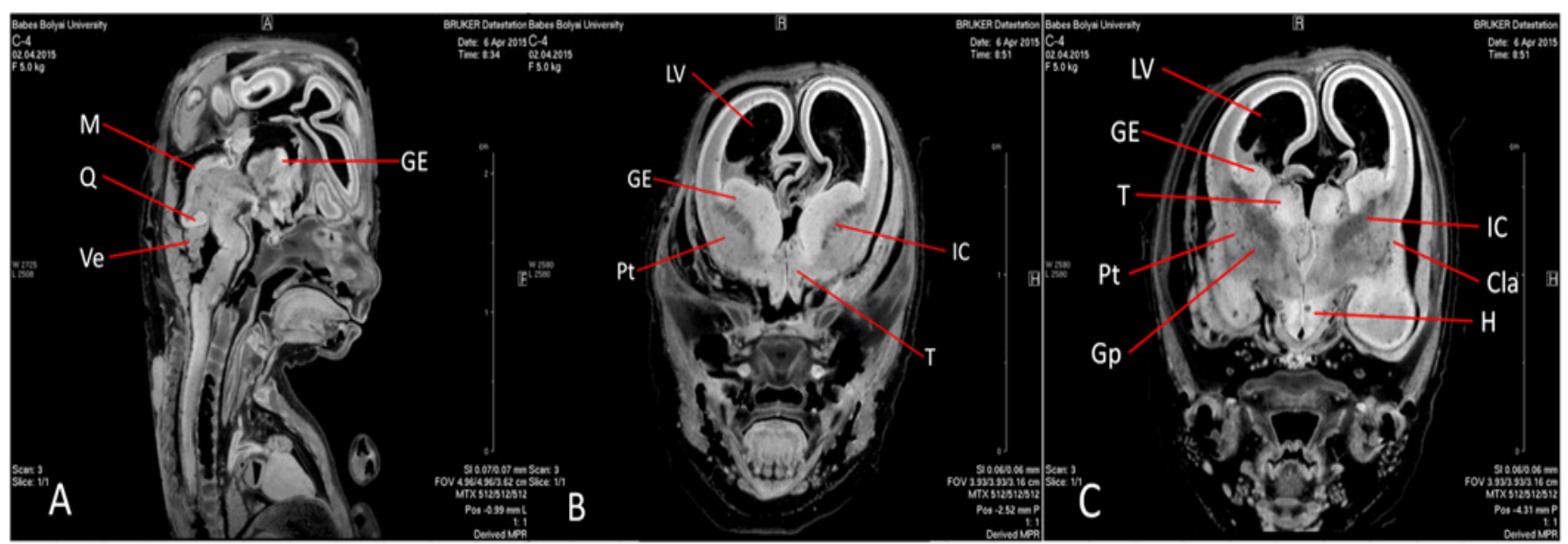

Figure 5. Human fetus $66 \mathrm{~mm}$ CRL, 13 GW. Sagittal (A) and coronal (B, C) sections are shown. M: mesencephalon, Q: quadrigeminal plate, Ve: cerebellar vermis, GE: ganglionic eminence, LV: lateral ventricle, Pt: putamen, T: thalamus, IC: internal capsule, Gp: globus pallidus, H: hypothalamus, Cla: claustrum.

In a $85 \mathrm{~mm}$ CRL fetus the developing brain has already a very complex structure. Details such as the anterior commissure and the inferior olivary nuclei can be observed. The GE is still well developed rostrally where it is adjacent to the anterior commissure but it flattens as the brain increases its dimensions. The basal nuclei excepting the caudate nucleus are separated from the GE by the fibers of the internal commissure (Figure 6).

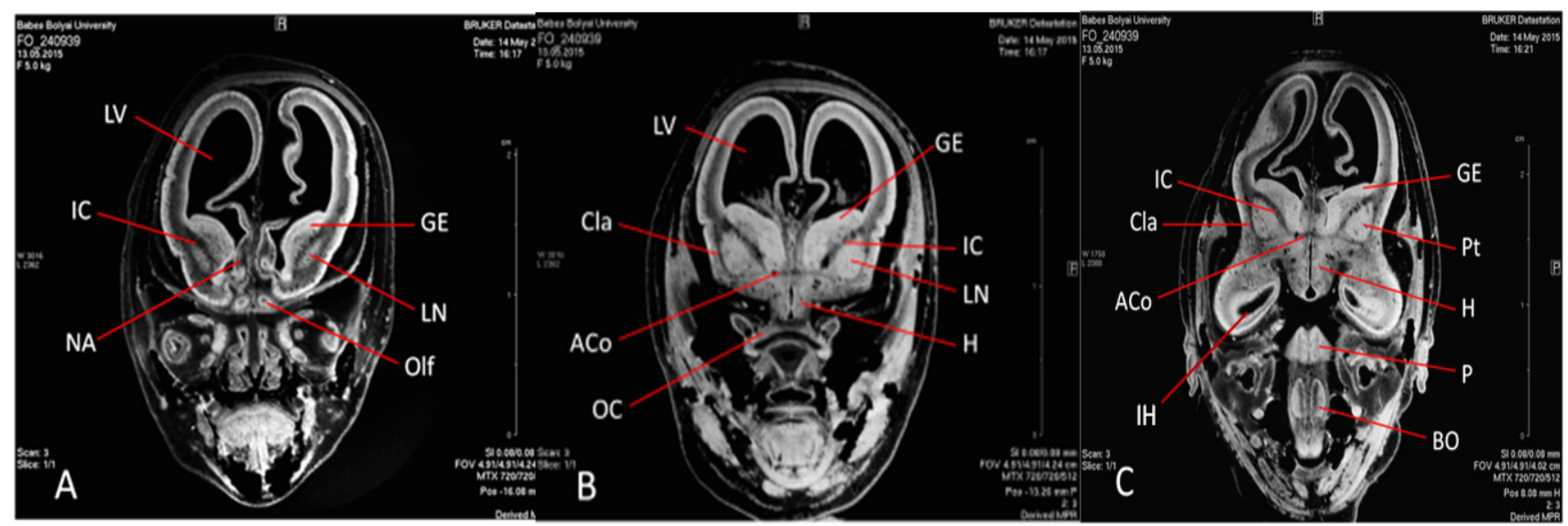

Figure 6. Human fetus $85 \mathrm{~mm}$ CRL, $14 \mathrm{GW}$. Coronal (A, B) and axial (C) sections are presented. LV: lateral ventricle, IC: internal capsule, NA: nucleus accumbens, Olf: olfactory bulbs, LN: lenticular nucleus, GE: ganglionic eminence, Cla: claustrum, ACo: anterior commissure, OC: optic chiasma, H: hypothalamus, BO: inferior olivary nucleus, P: pons, Pt: putamen, IH: inferior horn of the lateral ventricle. 
The structure of the ventral wall of the TV (subpallium) can be observed beginning with the $21 \mathrm{~mm}$ CRL embryo (Figure 7 panel A), while the lamination of the lateral wall of the TV (pallium, future neocortex) is evident beginning only with the $32 \mathrm{~mm}$ CRL fetus (Figure 7 panels B-F). At $10 \mathrm{GW}$ the lateral wall of the TV consists of three layers: internal layer which borders the lateral ventricle; intermediate layer and cortical plate respectively. The cortical plate and the internal layer provide a hyperintense signal while the intermediate layer is hypointense in T1wi protocol. Therefore, the wall of the TV appears as a sandwich-like structure.
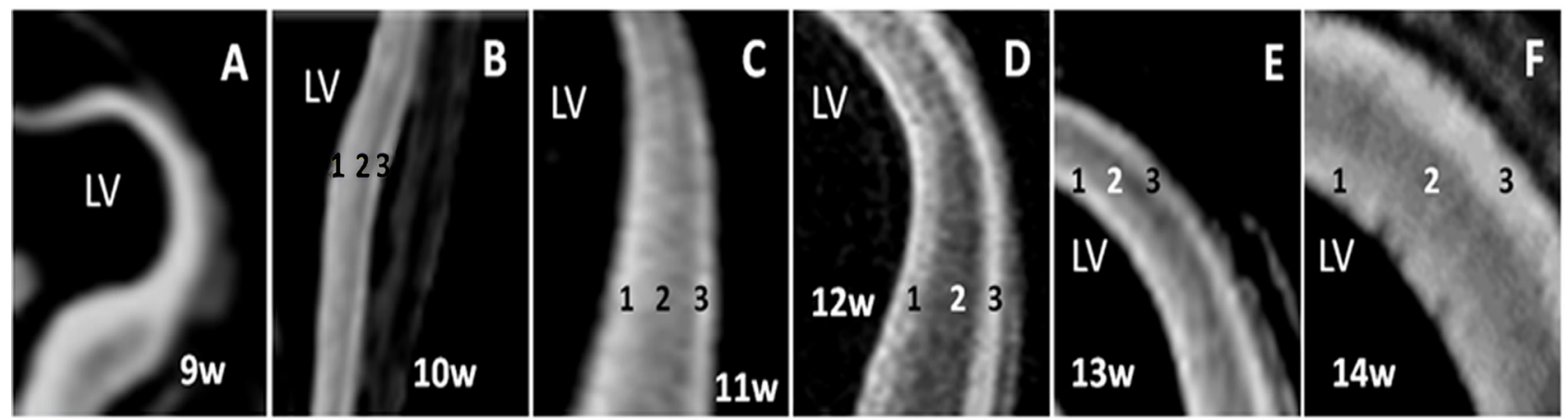

Figure 7. In panel A, which is a magnification of the image shown in Figure 1A, three layers can be noticed in the ventral wall of the TV (subpallium), the place where the ganglionic eminence will form. The structure of the lateral wall of the TV at different gestational ages is documented in panels B-F. 1: ventricular layer, 2: intermediate layer, 3: cortical plate, LV: lateral ventricle.

\section{Discussion}

The GE were depicted by using 7.04 Tesla microMRI in a series of six subjects ranging from $21 \mathrm{~mm}$ CRL $(9$ $\mathrm{GW})$ to $85 \mathrm{~mm}$ CRL (14 GW).

A major problem of the micro-MRI is the acquisition time, which, in our study, was of approximately 24 hours. This corresponds to the results of other researchers [16].

In T1-wi the GE appear as hyperintense images [17] located caudally and ventrally from the interventricular foramen. The hyperintense signal is due to the high cellular density, as well as to their vascularization which is richer than in any other region of the developing brain [18]. It was stated that the GE may be precisely depicted in the rostral part of the brain, where the width of the ventricular layer is important [19], but using micro-MRI the GE was clearly visualized in the caudal portion as well.

We noticed temporal and spatial changes in the morphology of the GE, which is different at different gestational ages, as the brain increases its dimensions and becomes more complex. Those changes reflect the dramatic morphological transformations of the developing brain, which are very fast especially in the first trimester of pregnancy. The shape and dimensions of the GE vary in different regions of the developing brain. The width of the GE decreases in a rostro-caudal direction: at the rostral end facing the future anterior horn of the lateral ventricle the GE is larger, while at the caudal end facing the well developed thalamus, the GE narrows. It was stated that the GEs are intersected twice in axial and coronal sections respectively because of their " $\mathrm{C}$ " shape which follows the expansion of the cerebral hemispheres [20], which was not observed in our sections.

Previous studies have demonstrated that three layers of the telencephalic walls can be depicted by using MRI: internal, subcortical and cortical zones [21]. Those zones correspond to seven histologic layers which are well known [22]. In our study the structure of the lateral wall of TV was depicted in a $32 \mathrm{~mm}$ CRL fetus (10 GW). The lamination of the ventral wall (subpallium) of TV was described earlier, in a $21 \mathrm{~mm}$ CRL embryo (9 GW), which was not found in literature. The subcortical zone is the most developed transitory layer of the encephalic wall during the fetal period [23].

The main advantage of micro-MRI is the accuracy of images, which is due to the high spatial resolution, almost similar to that obtained by histology [24]. The contributing factors to this outstanding spatial resolution are: lack of movements which avoids artifacts; high intensity of the magnetic field; long acquisition time. In our study we obtained a remarkable spatial resolution of $27 \mu \mathrm{m} /$ voxel, which allows visualization of tiny structures of the developing brain. Moreover, micro-MRI is reproducible and anytime multiple sections can be made through the 3D volume in order to better understand the spatial architecture 
of anatomical structures. Also, micro-MRI can supply 3D images of the brain by using 3D reconstruction methods.

Another advantage of micro-MRI when compared with other imaging methods like computed tomography is the excellent tissue contrast, which allows to precisely distinguish the different tissues [23], though astonishing details of the developing brain can be assessed. This method is non-invasive and it preserves the specimens which are available for further studies. Micro-MRI has become the standard method for in vitro morphological studies.

Even if the micro-MRI doesn't have a direct clinical application, it may provide incredibly accurate images for understanding the normal and pathological morphogenesis of the brain $[25,26]$. These images are useful for the assessment of the fetal brain and correct interpretation of the MRI images obtained in vivo at lower intensities of the magnetic field [24,27].

A limitation of our study consists of the small number of specimens, which is because human embryos and fetuses of good quality are difficult to obtain. Also, the cost of the method, which is very high for now, makes micro-MRI unaffordable for cohort studies.

\section{Conclusions}

Studies of embryology are still needed for a complete assessment of the developing brain, which can provide a better understanding of the normal and pathological morphogenesis of the central nervous system.

Micro-MRI offers to embryologists an alternative to the classical histological techniques. It is feasible for morphological studies of embryos and fetuses, providing accurate images due to the high spatial resolution and excellent tissue contrast. It is also reproducible and does not affect the integrity of the specimens which can be submitted to further studies.

Micro-MRI provides clear images of small nervous structures such as the GE. We can hope that improving the spatial resolutions of MRI machines by using more intense magnetic fields will provide "histological" images of the tissues, either in 2D slices or in 3D reconstructions.

\section{Acknowledgements}

This research was supported by the University of Medicine and Pharmacy Cluj-Napoca by the grant no. 4944.

\section{References}

1. Hansen DV, Lui JH, Flandin P, Yoshikawa K, Rubenstein JL, Alvarez-Buylla A, et al. Non-epithelial stem cells and cortical interneuron production in the human ganglionic eminences. Nat Neurosci. 2013;16:1576-1587.

2. O'Rahilly R, Müller F. The embryonic human brain. WileyLiss: New York; 1994: p. 15-18.
3. Müller F, O'Rahilly R. Embryonic Development of the Central Nervous System. In: Paxinos, G., Jürgen, K.M. (Ed.). The Human Nervous System, $2^{\text {nd }}$ Edition. Elsevier Academic Press: San Diego, California; 2004: p. 22-48.

4. Valiente M, Marin O. Neuronal migration mechanisms in development and disease. Curr Opin Neurobiol. 2010;20:6878.

5. ten Donkelaar HJ, Lammens M, Hori A. Clinical Neuroembryology: Development and Developmental Disorders of the Human Central Nervous System. Springer, Heidelberg; 2014: pp. 1-39.

6. Bandler RC, Mayer C, Fishell G. Cortical interneuron specification: the juncture of genes, time and geometry. Current Opinion in Neurobiology. 2017;42:17-24.

7. O'Rahilly R, Müller F. Significant features in the early prenatal development of the human brain. Ann Anat. 2008;190:105-118.

8. Matsuda Y, Ono S, Otake Y, Handa S, Kose K, Haishi T, et al. Imaging of a large collection of human embryo using a super-parallel MR microscope. Magn Reson Med Sci. 2007;6:139-146.

9. Lhuaire M, Martinez A, Kaplan H, Nuzillard JM, Renard Y, Tonnelet R, et al. Human developmental anatomy: Microscopic magnetic resonance imaging ( $\mu \mathrm{MRI})$ of four human embryos (from Carnegie Stage 10 to 20). Ann Anat. 2017;196:402-409.

10. Smith BR, Johnson GA, Groman EV, Linney E. Magnetic resonance microscopy of mouse embryos. Proc Natl Acad Sci U S A. 1994;91:3530-3533.

11. Smith BR. Visualizing human embryos. Sci Am. 1999;280:76-81.

12. Shiota K, Yamada S, Nakatsu-Komatsu T, Uwabe C, Kose K, Matsuda Y, et al. Visualization of human prenatal development by magnetic resonance imaging (MRI). Am J Med Genet A. 2007;143A:3121-3126.

13. Boitor-Borza D, Crivii C, Farcasanu S, Stamatian F. Morphology of the human brain in the embryonic period: anatomical study and assessment by $7-\mathrm{T}$ magnetic resonance imaging. Obstetrica si Ginecologia. 2015;63:47-52.

14. O'Rahilly R, Müller F. Developmental stages in human embryos: revised and new measurements. Cells Tissues Organs. 2010;192:73-84.

15. O'Rahilly R, Müller F. The Embryonic Human Brain: An Atlas of Developmental Stages, $3^{\text {rd }}$ edition. John Wiley \&Sons, Hohoken, New Jersey, USA 2006: p. 36-45.

16. Otake Y, Handa S, Kose K, Shiota K, Yamada S, Uwabe C. Magnetic resonance microscopy of chemically fixed human embryos at high spatial resolution. Magn Reson Med Sci. 2015;14:153-158.

17. Prayer D, Kasprian G, Krampl E, Ulm B, Witzani L, Prayer L, et al. MRI of normal fetal brain development. Eur J Radiol. 2006;57:199-216.

18. Ballabh P, Braun A, Nedergaard M. Anatomic analysis of blood vessels in germinal matrix, cerebral cortex, and white matter in developing infants. Pediatr Res. 2004;56:117-124.

19. Maas LC, Mukherjee P, Carballido-Gamio J, Veeraraghavan 
S, Miller SP, Partridge SC, et al. Early laminar organization of the human cerebrum demonstrated with diffusion tensor imaging in extremely premature infants. Neuroimage. 2004;22:1134-1140.

20. Ulfig N. The ganglionic eminence--a putative intermediate target of amygdaloid connections. Developmental Brain Res. 2003;139:313-318.

21. Huang H, Jeon T, Sedmak G, Pletikos M, Vasung L, Xu X, et al. Coupling diffusion imaging with histological and gene expression analysis to examine the dynamics of cortical areas across the fetal period of human brain development. Cereb Cortex. 2013;23:2620-2631.

22. Bystron I, Blakemore C, Rakic P. Development of the human cerebral cortex: boulder committee revisited. Nat Rev Neurosci. 2008;9:110-122.

23. Zhan J, Dinov ID, Li J, Zhang Z, Hobel S, Shi Y, et al. Spatial- temporal atlas of human fetal brain development during the early second trimester. Neuroimage. 2013;82:115-126.

24. Lin $X$, Zhanga Z, Teng G, Meng H, Yu T, Hou Z, et al. Measurements using 7.0 T post-mortem magnetic resonance imaging of the scalar dimensions of the fetal brain between 12 and 20 weeks gestational age. Int $\mathrm{J}$ Dev Neurosci. 2011;29:885-889.

25. Bendersky M, Musolino PL, Rugilo C, Schuster G, Sica RE. Normal anatomy of the developing fetal brain. Ex vivo anatomical-magnetic resonance imaging correlation. J Neurol Sci. 2006;250:20-26.

26. Al-Mukhtar A, Kasprian G, Schmook MT, Brugger PC, Prayer D. Diagnostic pitfalls in fetal brain MRI. Semin Perinatol. 2009;33:251-258.

27. Glenn OA. Normal development of the fetal brain by MRI. Semin Perinatol. 2009;33:208-219. 